Journal of Animal and Veterinary Advances 10 (15): 1927-1933, 2011

ISSN: $1680-5593$

(C) Medwell Journals, 2011

\title{
Protein Requirement of Thai Native Cattle Compared with Meta-Analysis of Bos indicus Cattle
}

\author{
P. Paengkoum, S. Thongruand, K. Kamkajon and S. Paengkoum \\ Faculty of Animal Sciences and Agricultural Technology, \\ Silpakorn University, Phetchaburi IT Campus, Cha-Um, \\ 76120 Phetchaburi, Thailand
}

\begin{abstract}
The objective of this study was to determine protein requirement of Thai native cattle compared with the meta-analysis of Bos indicus. The 24 Thai native cattle with body weight of $134+11.2 \mathrm{~kg}$ were arranged in a randomized block design with 8 replications and 3 treatments. Cattle were fed with total mixed ratio consisted 3 levels of protein $(6.0,9.0$ and $12.0 \%)$. The meta-analys is of protein requirement for maintenance and for growth of Thai native and Brahman cattle were used to compare with the experiment. A database from 48 observations of trials conducted under diverse feeding condition in Thailand was subjected to regression analysis using a linear model. Dry matter, organic mater, crude protein and Neutral Detergent Fiber (NDF) digestibility, N absorption and $\mathrm{N}$ retention increased $(\mathrm{p}<0.05)$ with increasing levels of $\mathrm{CP}$. Prediction equation $\mathrm{CP}$ intake (CPI, gCP kg ${ }^{-1} \mathrm{BW}^{0.75}$ ) with relation to Average Daily Gain ( $\mathrm{ADG}, \mathrm{g} \mathrm{kg}^{-1} \mathrm{BW}^{0.75}$ ) was $\mathrm{CPI}=0.413+4.28$ $\left(\mathrm{R}^{2}=0.896, \mathrm{SE}=0.774, \mathrm{p}<0.05, \mathrm{n}=24\right)$. It can be explained that the CP for maintenance of Thai native cattle is $4.28 \mathrm{gCP} \mathrm{kg}^{-1} \mathrm{BW}^{0.75}$. Results from the meta-analysis, the relation between Nitrogen Intake $\left(\mathrm{NI}, \mathrm{g} \mathrm{kg}^{-1} \mathrm{BW}^{0.75}\right)$ and Nitrogen Retention $\left(\mathrm{NR}, \mathrm{g} \mathrm{kg}^{-1} \mathrm{BW}^{0.75}\right)$ of Thai native were $\mathrm{NR}=-0.41(\mathrm{SE}=0.16)+0.58(\mathrm{SE}=0.10) \mathrm{NI}$ $\left(\mathrm{n}=26, \mathrm{R}^{2}=0.57, \mathrm{RSD}=0.27, \mathrm{p}<0.05\right)$ and Brahman were $\mathrm{NR}=-0.34(\mathrm{SE}=0.1)+0.70(\mathrm{SE}=0.04) \mathrm{NI}(\mathrm{n}=14$, $\mathrm{R}^{2}=0.96, \mathrm{RSD}=0.20, \mathrm{p}<0.05$ ). The relation between NI and Dry Matte Digestibility (DMD, \%) for Thai native were $\mathrm{DMD}=48.90(\mathrm{SE}=4.92)+11.10(\mathrm{SE}=3.02) \mathrm{NI}\left(\mathrm{n}=12, \mathrm{R}^{2}=0.57, \mathrm{RSD}=4.90, \mathrm{p}<0.05\right)$ and Brahman were $\mathrm{DMD}=48.55(\mathrm{SE}=6.44)+7.16(\mathrm{SE}=1.94) \mathrm{NI}\left(\mathrm{n}=11, \mathrm{R}^{2}=0.60, \mathrm{RSD}=7.47, \mathrm{p}<0.05\right)$. The relation between $\mathrm{NI}$ and Organic Matter Digestibility $(\mathrm{OMD}, \%)$ for Thai native were $\mathrm{OMD}=56.68(\mathrm{SE}=2.75)+5.27(\mathrm{SE}=1.72) \mathrm{NI}$ $\left(\mathrm{n}=12, \mathrm{R}^{2}=0.48, \mathrm{RSD}=2.78, \mathrm{p}<0.05\right)$, Brahman were $\mathrm{OMD}=50.79(\mathrm{SE}=3.75)+6.18(\mathrm{SE}=1.13) \mathrm{NI}(\mathrm{n}=11$, $\left.\mathrm{R}^{2}=0.77, \mathrm{RSD}=4.35, \mathrm{p}<0.05\right)$. The relation between NI and NDF Digestibility (NDFD, \%) for Thai native were $\mathrm{NDFD}=50.94(\mathrm{SE}=1.16)+4.26(\mathrm{SE}=0.70) \mathrm{NI}\left(\mathrm{n}=8, \mathrm{R}^{2}=0.86, \mathrm{RSD}=1.01, \mathrm{p}<0.05\right)$, Brahman were NDFD $=51.52$ $(\mathrm{SE}=2.80)+5.60(\mathrm{SE}=0.79) \mathrm{NI}\left(\mathrm{n}=9, \mathrm{R}^{2}=0.88, \mathrm{RSD}=2.80, \mathrm{p}<0.05\right)$. The relationship between $\mathrm{NI}$ and $\mathrm{CP}$ digestibility $(\mathrm{CPD}, \%)$ for Thai native were $\mathrm{CPD}=47.86(\mathrm{SE}=4.50)+13.09(\mathrm{SE}=2.76) \mathrm{NI}\left(\mathrm{n}=11, \mathrm{R}^{2}=0.71\right.$, $\mathrm{RSD}=4.29, \mathrm{p}<0.05)$. The relationship between NI and Blood Urea Nitrogen (BUN, $\mathrm{mg} \%$ ) for Thai native were $\mathrm{BUN}=7.56(\mathrm{SE}=1.36)+0.09(\mathrm{SE}=0.11) \mathrm{NI}\left(\mathrm{n}=9, \mathrm{R}^{2}=0.08, \mathrm{RSD}=2.86, \mathrm{p}<0.05\right)$, Brahman were $\mathrm{BUN}=14.17$ $(\mathrm{SE}=3.04)-1.21(\mathrm{SE}=0.95) \mathrm{NI}\left(\mathrm{n}=4, \mathrm{R}^{2}=0.45, \mathrm{RSD}=2.75, \mathrm{p}<0.05\right)$. The relationship between $\mathrm{NI}$ and ruminal Ammonia Nitrogen $\left(\mathrm{NH}_{3} \mathrm{~N}, \mathrm{mg} \%\right)$ for Thai native were $\mathrm{NH}_{3} \mathrm{~N}=-7.99(\mathrm{SE}=3.07)+12.14(\mathrm{SE}=1.84) \mathrm{NI}$ $\left(\mathrm{n}=7, \mathrm{R}^{2}=0.90, \mathrm{RSD}=1.56, \mathrm{p}<0.05\right.$. The results indicated that protein requirement by nitrogen intake of Thai native and Brahman have relation to nitrogen retention, $\mathrm{DMD}, \mathrm{OMD}, \mathrm{NDFD}, \mathrm{ADFD}, \mathrm{CPD}, \mathrm{BUN}$ and $\mathrm{NH}_{3} \mathrm{~N}$ but the potential of prediction were indicated to $\mathrm{R}^{2}$ value.
\end{abstract}

Key words: Protein requirement, nitrogen intake, Thai native cattle, meta-analysis, composition, body tissues

\section{INTRODUCTION}

The Bos indicus cattle and their crossbreds including Thai native are commonly used in beef production systems in tropical regions. They possess abilities to withstand hot and humid weather to tolerate intense sunshine to resist parasites and to utilize poor quality forages (Turner, 1980). The National Research Council guidelines for beef cattle production (NRC, 2000) are widely adopted to formulate diets around the world however, protein and nutrient requirements are based on Bos taurus data. The NRC (2000) indicates that Bos indicus breeds require about $10 \%$ less $\mathrm{NE}_{\mathrm{m}}$ than beef breeds of Bos taurus. Additionally, it has been recognized

Corresponding Author: P. Paengkoum, School of Animal Production Technology, Institute of Agricultural Technology, Suranaree University of Technology, Muang, 30000 Nakhon Ratchasima, Thailand 
that sex and castrate status influences growth of body tissues, affecting carcass composition and feed efficiency (Berg and Butterfield, 1976) and the $\mathrm{NE}_{\mathrm{m}}$ requirement (ARC, 1980; NRC, 2000). Therefore, improvements in the beef cattle production in tropical and subtropical regions require an accurate assessment of protein requirements of cattle compare to the protein requirement of beef cattle in temperate. The objective of this study was to perform a meta-analysis to determine protein requirements for maintenance and growth of Bos indicus beef cattle and Thai native beef cattle from independent studies.

\section{MATERIALS AND METHODS}

Animals and management: The 24 male Thai native beef cattle (Bos indicus) with body weight of $134(\mathrm{SD} \pm 11.2) \mathrm{kg}$ were kept in individual pens and arranged in a Randomized Complete Block Design (RCBD) with 8 replications (blocks) and 3 treatments. Cattle were allowed an adjustment period of 2 weeks and treated against anthelmintics and intestinal parasites using Ivermectin. Cattle were fed dietary Crude Protein (CP) levels of 6.0, 9.0 and $12.0 \% \mathrm{CP}$ with similar amounts of Metabolizable Energy (ME) in Total Mixed Ratio (TMR). The total feed intake was fixed at $2.5 \%$ of body weight. The daily ratios were offered to the animals in two equal portions at 0830 and $1530 \mathrm{~h}$. Refusals were weighed daily prior to the morning feeding to determined daily Dry Matter Intake (DMI). Body weight of each animal was measured twice monthly immediately before the morning feeding. Drinking water was freely available. The experiment consisted of 14 weeks, 2 weeks of adaptation following by 12 weeks of experimental or feeding periods. The last week of feeding period consisted of 2 days of adaptation to the metabolic crates, 7 days of digestibility and $\mathrm{N}$ balance studies. Samples of feed refusal, feces and urine were collected before feeding morning to determine digestibility and $\mathrm{N}$ balance. Daily fecal output of each cattle was measured and a $10 \%$ sub-sample collected and stored at $-20^{\circ} \mathrm{C}$. The samples were dried $\left(60^{\circ} \mathrm{C}\right)$, ground through $1 \mathrm{~mm}$ sieve and stored for chemical analysis. Daily urine output was collected into a plastic container, $10 \%$ of the urine were later sampled and frozen and stored at $-20^{\circ} \mathrm{C}$ until the analysis for energy and $\mathrm{N}$ contents. Representative samples of feed and feces collected during the digestibility trial were analyzed according to AOAC (1984), ash and CP and fiber components (Van Soest et al., 1991). Apparent digestion coefficients were calculated using equations of Schneider and Flatt (1975).
Statistics: A general linear model and correlation were used to evaluate the relationship between crude protein or Nitrogen $(\mathrm{N})$ intake and their excretion via feces and urine. The data was analyzed by the general linear models procedure of the Statistical Analysis System Institute SAS (1999). Using Duncan's New Multiple Range test (Steel and Torries, 1980) compare treatment means.

A meta-analysis: A database including 48 observations from 12 feeding trials of Bos indicus: Brahman and Thai native in Thailand. Constructed and analyzed to determine crude protein requirements for maintenance and for growth or gain ( 7 observations of Thai native and 5 observations of Brahman) using mixed linear model (SAS, 1999) by regressing nitrogen intake $\left(\mathrm{gN} \mathrm{kg}^{-1} \mathrm{BW}^{0.75}\right.$ day $\left.^{-1}\right)$ against nitrogen retention $\left(\mathrm{gN} \mathrm{kg}^{-1} \mathrm{BW}^{0.75}\right.$ day $\left.^{-1}\right)$ according to St-Pierre (2001):

$$
\begin{aligned}
& \mathrm{Y}_{\mathrm{ij}}=\mathrm{B}_{0}+\mathrm{B}_{1} \mathrm{X}_{\mathrm{ij}}+\mathrm{s}_{\mathrm{i}}{ }^{*}+\mathrm{b}_{\mathrm{i}}{ }^{*} \mathrm{X}_{\mathrm{ij}}+\mathrm{e}_{\mathrm{ij}} \\
& \mathrm{Y}=\text { The expected outcome for CPI } \\
& \mathrm{B}_{0}+\mathrm{B}_{1} \mathrm{X}_{\mathrm{ij}} \quad=\text { Fixed effect part of model } \\
& \mathrm{s}_{\mathrm{i}}^{*}+\mathrm{b}_{\mathrm{i}}^{*} \mathrm{X}_{\mathrm{ij}}+\mathrm{e}_{\mathrm{ij}}=\text { Random effect part of model }
\end{aligned}
$$

Performance of the derived prediction equation was tested by calculating predicted values for each dataset using the prediction models and comparing those to the actual values. Degree of over or under prediction was expressed as mean proportion bias (\%) which can calculated as the slope of the regression of actual on predicted values at zero intercept according to Mandal et al. (2005) and accuracy of prediction was analyzed using mean prediction error. Model prediction was evaluated for accuracy by paired t-test of actual and predicted values. A non-significant $(\mathrm{p}>0.05)$ paired t-test between actual and predicted values indicated good match between values calculated using the derived prediction model and actual values (Paul et al., 2003).

\section{RESULTS AND DISCUSSION}

Publications of database used in protein requirement for maintenance and gain estimation are shown in Table 1. Total dry matter feed intake, nutrients digestibility and ADG in cattle fed with different levels of CP are shown in Table 2. There were no effects of crude protein levels on total dry matter intake $(\mathrm{kg}, \mathrm{BW} \%$ and $\mathrm{g} \mathrm{kg}^{-1} \mathrm{BW}^{0.75}$ ). Dry matter, OM, CP and NDF digestibility in cattle fed with $12 \% \mathrm{CP}$ was the highest and significantly higher $(\mathrm{p}<0.05)$ than those fed 9 and $6 \% \mathrm{CP}$. Moreover, all nutrients digestibility of cattle fed with $9 \%$ CP was significantly higher $(\mathrm{p}<0.05)$ than that of cattle fed with 6\% CP (Table 3). 
Table 1: Publications of database used in protein requirement for maintenance and gain estimation

\begin{tabular}{|c|c|c|c|c|}
\hline References & Breed & $\mathrm{N}$ & $\begin{array}{c}\text { Body } \\
\text { weight }(\mathrm{kg})\end{array}$ & $\begin{array}{c}\text { DM intake }\left(\mathrm{g} \mathrm{B}^{-}\right. \\
\left.\mathrm{Wkg}^{0.75} \mathrm{day}^{-1}\right)\end{array}$ \\
\hline Thai Native beef cattle & & & & \\
\hline $\begin{array}{l}\text { Chantiratikul and } \\
\text { Chumpawadee (2009) }\end{array}$ & Thai native & 4 & 132.00 & 64.43 \\
\hline $\begin{array}{l}\text { Paengkoum and } \\
\text { Tatsapong (2009) }\end{array}$ & Thai native & 8 & 104.00 & 90.53 \\
\hline Senarath et al. (2009) & Thai native & 4 & 105.00 & 49.65 \\
\hline Otsuka et al. (2010) & Thai native & 4 & 132.00 & 21.12 \\
\hline Paengkoum (2010) & Thai native & 18 & 125.00 & 84.30 \\
\hline Shengchang et al. (2010) & Thai native & 6 & 154.00 & 80.18 \\
\hline Paengkoum (2010) & Thai native & 4 & 104.00 & 80.55 \\
\hline Brahman cattle & & & & \\
\hline $\begin{array}{l}\text { Khuamankgorn et al. } \\
\text { (2009) }\end{array}$ & Brahman & 4 & 350.00 & 68.04 \\
\hline Chaokaur et al. (2009) & Brahman & 16 & 343.94 & 36.48 \\
\hline Chaokaur et al. (2009) & Brahman & 16 & 276.47 & 35.98 \\
\hline Yuangklang (2009) & Brahman & 8 & 204.50 & 124.49 \\
\hline Kawashima et al. (2006) & Brahman & 4 & 336.00 & 52.23 \\
\hline
\end{tabular}

Table 2: Effect of crude protein levels on feed intake, digestibility, ruminal ammonia-nitrogen $\left(\mathrm{NH}_{3}-\mathrm{N}\right)$, Blood Urea Nitrogen (BUN) and Average Daily Gain (ADG) of Thai native cattle

\begin{tabular}{|c|c|c|c|c|}
\hline \multirow[b]{2}{*}{ Variables } & \multicolumn{3}{|c|}{ Dietary protein $(\%)$} & \multirow[b]{2}{*}{ SEM } \\
\hline & 6.0 & 9.0 & 12.0 & \\
\hline \multicolumn{5}{|l|}{ Dry matter intake } \\
\hline $\mathrm{kg} \mathrm{day}^{-1}$ & 4.10 & 4.00 & 4.10 & 0.05 \\
\hline BW\% & 3.10 & 3.00 & 3.10 & 0.03 \\
\hline $\mathrm{g} \mathrm{kg}^{-1} \mathrm{BW}^{0.75}$ & 103.80 & 102.30 & 105.70 & 1.00 \\
\hline \multicolumn{5}{|l|}{ Digestibility (\%) } \\
\hline Dry matter & $58.60^{c}$ & $64.50^{b}$ & $69.20^{\mathrm{a}}$ & 1.14 \\
\hline Organic matter & $59.80^{c}$ & $65.70^{b}$ & $70.60^{\mathrm{a}}$ & 1.17 \\
\hline Crude protein & $55.20^{\mathrm{c}}$ & $66.80^{b}$ & $68.90^{\mathrm{a}}$ & 1.22 \\
\hline Neutral detergent fiber & $54.60^{c}$ & $58.70^{b}$ & $62.30^{\mathrm{a}}$ & 1.18 \\
\hline Ruminal $\mathrm{NH}_{3}-\mathrm{N}(\mathrm{mg} \%)$ & $7.80^{\mathrm{c}}$ & $12.70^{b}$ & $15.60^{\mathrm{a}}$ & 0.87 \\
\hline Blood urea nitrogen (mg\%) & $10.30^{c}$ & $15.80^{b}$ & $17.70^{\mathrm{a}}$ & 0.76 \\
\hline Average daily gain $\left(\mathrm{kg} \mathrm{day}^{-1}\right)$ & $0.05^{c}$ & $0.10^{b}$ & $0.15^{\mathrm{a}}$ & 0.08 \\
\hline
\end{tabular}

${ }^{\mathrm{a} c} \mathrm{~V}$ Values on the same row under each main effect with different superscripts differ significantly $(\mathrm{p}<0.05)$

Table 3: Summary database for prediction of protein requirements for maintenance and for gain

\begin{tabular}{|c|c|c|c|c|}
\hline Item & $\mathrm{n}$ & Mean & Minimum & Maximum \\
\hline \multicolumn{5}{|l|}{ Thai Native cattle } \\
\hline Body weight (kg) & 48 & 122.290 & 104.000 & 154.00 \\
\hline $\mathrm{N}$ intake $\left(\mathrm{N} \mathrm{kg}^{-1} \mathrm{BW}^{0.75} \mathrm{day}^{-1}\right)$ & 48 & 1.420 & 0.600 & 2.32 \\
\hline $\mathrm{N}$ retained $\left(\mathrm{g} \mathrm{B}^{-1} \mathrm{Wkg}^{0.75}\right)$ & 48 & 0.410 & -0.180 & 13.17 \\
\hline DM digestibility (\%) & 36 & 66.380 & 54.660 & 75.83 \\
\hline OM digestibility (\%) & 36 & 65.070 & 57.050 & 70.07 \\
\hline CP digestibility (\%) & 30 & 68.340 & 53.600 & 81.88 \\
\hline NDF digestibility (\%) & 36 & 59.020 & 52.300 & 66.00 \\
\hline ADF digestibility (\%) & 10 & 51.970 & 46.250 & 61.05 \\
\hline $\mathrm{pH}$ & 18 & 6.750 & 6.460 & 7.07 \\
\hline $\mathrm{NH}_{3} \mathrm{~N}(\mathrm{mg} \%)$ & 18 & 10.880 & 6.550 & 19.77 \\
\hline Blood urea nitrogen (mg\%) & 8 & 20.540 & 2.810 & 12.38 \\
\hline \multicolumn{5}{|l|}{ Brahman cattle } \\
\hline Body weight (kg) & 48 & 285.895 & 276.470 & 350.00 \\
\hline $\mathrm{N}$ intake $\left(\mathrm{N} \mathrm{kg}^{-1} \mathrm{BW}^{0.75}\right.$ day $\left.^{-1}\right)$ & 48 & 2.380 & 0.435 & 4.63 \\
\hline $\mathrm{N}$ retained $\left(\mathrm{g} \mathrm{B}^{-1} \mathrm{Wkg}^{0.75}\right)$ & 44 & 0.940 & -1.950 & 2.89 \\
\hline DM digestibility (\%) & 16 & 70.850 & 49.600 & 80.90 \\
\hline OM digestibility (\%) & 16 & 70.040 & 51.800 & 75.20 \\
\hline NDF digestibility (\%) & 12 & 70.130 & 51.800 & 76.10 \\
\hline ADF digestibility (\%) & 12 & 57.120 & 42.900 & 59.80 \\
\hline Blood urea nitrogen (mg\%) & 8 & 9.670 & 5.500 & 14.48 \\
\hline
\end{tabular}

The relation between nitrogen intake (NI, $\mathrm{g} \mathrm{kg}^{-1}$ $\mathrm{BW}^{0.75}$ ) and nitrogen retention $\left(\mathrm{NR}, \mathrm{g} \mathrm{kg}^{-1} \mathrm{BW}^{0.75}\right)$ for
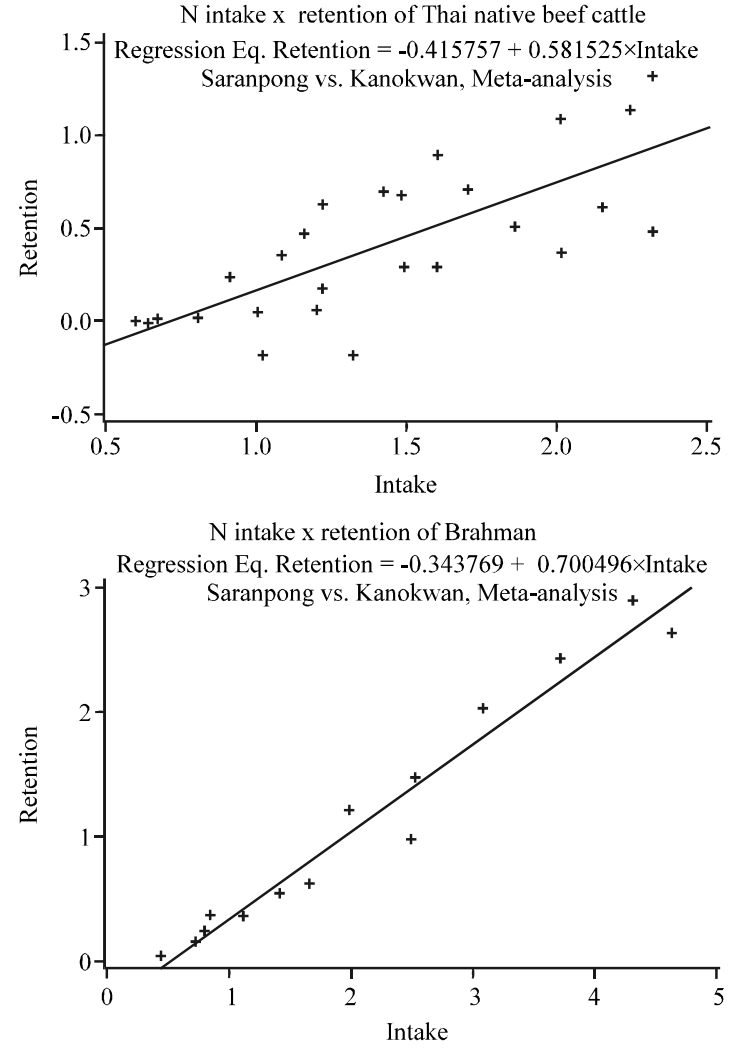

Fig. 1: Relationship between Nitrogen Intake (NI, $\mathrm{g} \mathrm{kg}^{-1}$ $\mathrm{BW}^{0.75}$ ) and Nitrogen Retention ( $\mathrm{NR}, \mathrm{g} \mathrm{kg}^{-1} \mathrm{BW}^{0.75}$ ) for Thai native beef cattle (a) describes equation; $\mathrm{NR}=-0.42(\mathrm{SE}=0.16)+0.58(\mathrm{SE}=0.10) \mathrm{NI}(\mathrm{n}=26$, $\left.\mathrm{R}^{2}=0.57, \mathrm{RSD}=0.27, \mathrm{p}<0.01\right)$, Brahman cattle (b) describes equation; $\mathrm{NR}=-0.34(\mathrm{SE}=0.1)+0.70$ $(\mathrm{SE}=0.04) \mathrm{NI}\left(\mathrm{n}=14, \mathrm{R}^{2}=0.96, \mathrm{RSD}=0.20, \mathrm{p}<0.01\right)$

Thai native were $\mathrm{NR}=-0.41_{(\mathrm{SE}=0.16)}+0.58_{(\mathrm{SE}=0.10)} \mathrm{NI}(\mathrm{n}=26$, $\left.\mathrm{R}^{2}=0.57, \mathrm{RSD}=0.27, \mathrm{p}<0.01\right)$, Brahman were $\mathrm{NR}=-0.34_{(\mathrm{SE}=0.1)}+0.70_{(\mathrm{SE}=0.04)} \mathrm{NI}\left(\mathrm{n}=14, \mathrm{R}^{2}=0.96\right.$, $\mathrm{RSD}=0.20, \mathrm{p}<0.01$ ) (Fig. 1). The relation between NI and Dry Matte Digestibility (DMD\%) for Thai native were $\mathrm{DMD}=48.90_{(\mathrm{SE}=4.92)}+11.10_{(\mathrm{SE}=3.02)} \mathrm{NI}\left(\mathrm{n}=12, \mathrm{R}^{2}=0.57\right.$, $\mathrm{RSD}=4.90, \mathrm{p}<0.01)$, Brahman were $\mathrm{DMD}=48.55_{(\mathrm{SE}=6.44)}+$ $7.16_{(\mathrm{SE}=1.94)} \mathrm{NI}\left(\mathrm{n}=11, \mathrm{R}^{2}=0.60, \mathrm{RSD}=7.47, \mathrm{p}<0.01\right)$ (Fig. 2).

The relation between NI and Organic Matter Digestibility $(\mathrm{OMD}, \%)$ for Thai native were $\mathrm{OMD}=$ $56.68_{(\mathrm{SE}=2.75)}+5.27_{(\mathrm{SE}=1.72)} \mathrm{NI}\left(\mathrm{n}=12, \mathrm{R}^{2}=0.48, \mathrm{RSD}=2.78\right.$, $\mathrm{p}<0.01)$, Brahman were $\mathrm{OMD}=50.79_{(\mathrm{SE}=3.75)}+6.18_{(\mathrm{SE}=1.13)}$ NI ( $\mathrm{n}=11, \mathrm{R}^{2}=0.77, \mathrm{RSD}=4.35, \mathrm{p}<0.01$ ) (Fig. 3). The relation between NI and NDF digestibility (NDFD, \%) for Thai native were NDFD $=50.94_{(\mathrm{SE}=1.16)}+4.26_{(\mathrm{SE}=0.70)} \mathrm{NI}$ $\left(\mathrm{n}=8, \mathrm{R}^{2}=0.86, \mathrm{RSD}=1.01, \mathrm{p}<0.01\right)$, Brahman were $\mathrm{NDFD}=51.52_{(\mathrm{SE}=2.80)}+5.60_{(\mathrm{SE}=0.79)} \mathrm{NI}\left(\mathrm{n}=9, \mathrm{R}^{2}=0.88\right.$, 

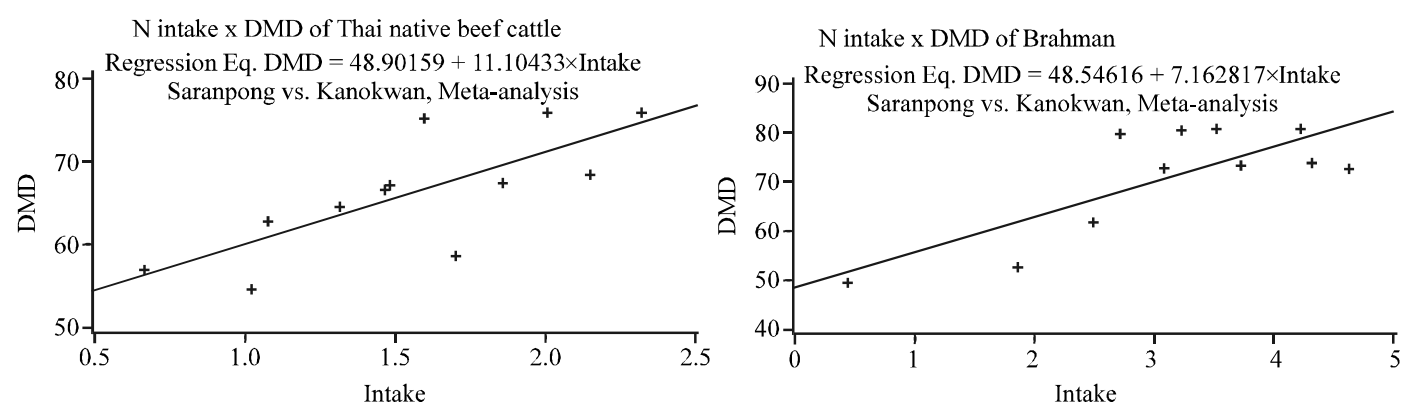

Fig. 2: Relationship between Nitrogen Intake $\left(\mathrm{NI}, \mathrm{g} \mathrm{kg}^{-1} \mathrm{BW}^{0.75}\right)$ and $\mathrm{DM}$ Digestibility (DMD\%) for Thai native beef cattle (a) describes equation; $\mathrm{DMD}=48.90(\mathrm{SE}=4.92)+11.10(\mathrm{SE}=3.02) \mathrm{NI}\left(\mathrm{n}=12, \mathrm{R}^{2}=0.57, \mathrm{RSD}=4.90, \mathrm{p}<0.01\right)$, Brahman cattle (b) describes equation: $\mathrm{DMD}=48.55(\mathrm{SE}=6.44)+7.16(\mathrm{SE}=1.94) \mathrm{NI}\left(\mathrm{n}=11, \mathrm{R}^{2}=0.60, \mathrm{RSD}=7.47\right.$, $\mathrm{p}<0.01$ )
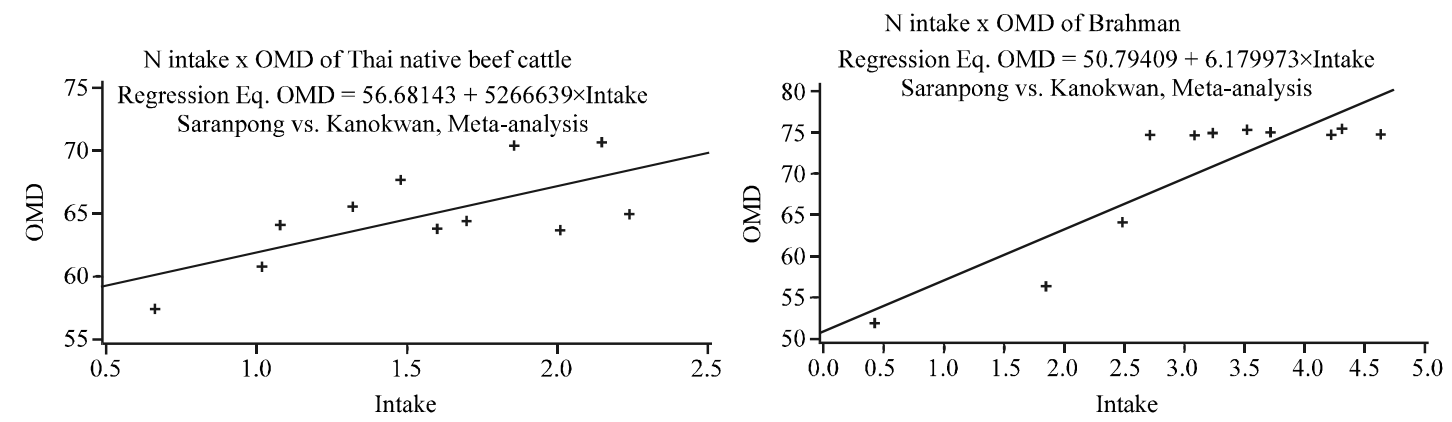

Fig. 3: Relationship between Nitrogen Intake $\left(\mathrm{NI}, \mathrm{g} \mathrm{kg}^{-1} \mathrm{BW}^{0.75}\right)$ and $\mathrm{OM}$ Digestibility $(\mathrm{OMD} \%)$ for Thai native beef cattle (a) describes equation; $\mathrm{OMD}=56.68(\mathrm{SE}=2.75)+5.27(\mathrm{SE}=1.72) \mathrm{NI}\left(\mathrm{n}=12, \mathrm{R}^{2}=0.48, \mathrm{RSD}=2.78, \mathrm{p}<0.01\right)$, Brahman cattle $(b)$ describes equation; $\mathrm{OMD}=50.79(\mathrm{SE}=3.75)+6.18(\mathrm{SE}=1.13) \mathrm{NI}\left(\mathrm{n}=11, \mathrm{R}^{2}=0.77, \mathrm{RSD}=4.35\right.$, $\mathrm{p}<0.01$ )
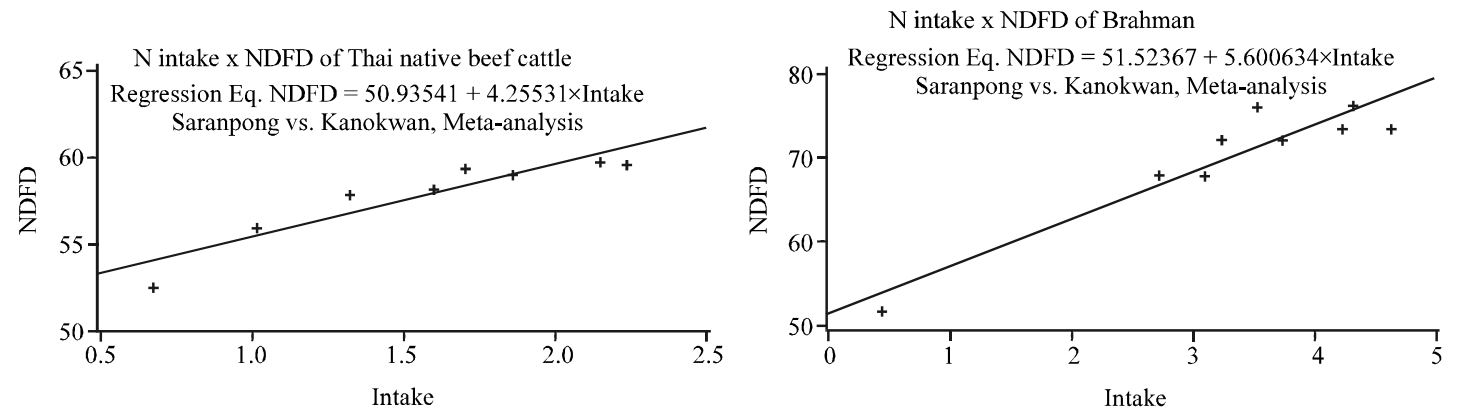

Fig. 4: Relationship between Nitrogen Intake (NI, $\mathrm{g} \mathrm{kg}^{-1} \mathrm{BW}^{0.75}$ ) and NDF Digestibility (NDFD\%) for Thai native beef cattle (a) describes equation: $\mathrm{NDFD}=50.94(\mathrm{SE}=1.16)+4.26(\mathrm{SE}=0.70) \mathrm{NI}\left(\mathrm{n}=8, \mathrm{R}^{2}=0.86, \mathrm{RSD}=1.01, \mathrm{p}<0.01\right)$, Brahman cattle $(\mathrm{b})$ describes equation: $\mathrm{NDFD}=51.52(\mathrm{SE}=2.80)+5.60(\mathrm{SE}=0.79) \mathrm{NI}\left(\mathrm{n}=9, \mathrm{R}^{2}=0.88, \mathrm{RSD}=2.80\right.$, $\mathrm{p}<0.01$ )

$\mathrm{RSD}=2.80, \mathrm{p}<0.01)$ (Fig. 4). The relation between NI and ADF Digestibility $(A D F D \%)$ for Thai native were $\mathrm{ADFD}=68.73_{(\mathrm{SE}=5.31)}-8.55_{(\mathrm{SE}=2.81)} \mathrm{NI}\left(\mathrm{n}=6, \mathrm{R}^{2}=0.70\right.$, $\mathrm{RSD}=2.63, \mathrm{p}<0.01)$, Brahman were $\mathrm{ADFD}=44.59_{(\mathrm{SE}=2.80)}$ $+3.77_{(\mathrm{SE}=0.79)} \mathrm{NI}\left(\mathrm{n}=9, \mathrm{R}^{2}=0.76, \mathrm{RSD}=2.81, \mathrm{p}<0.01\right)$
(Fig. 5). The relationship between NI and CP Digestibility $(\mathrm{CPD}, \%)$ for Thai native were $\mathrm{CPD}=47.86_{\text {(SE }=4.50)}+$ $13.09_{(\mathrm{SE}=2.76)} \mathrm{NI}\left(\mathrm{n}=11, \mathrm{R}^{2}=0.71, \mathrm{RSD}=4.29, \mathrm{p}<0.01\right)$ (Fig. 6). The relationship between NI and Blood Urea Nitrogen (BUN, mg\%) for Thai native were 

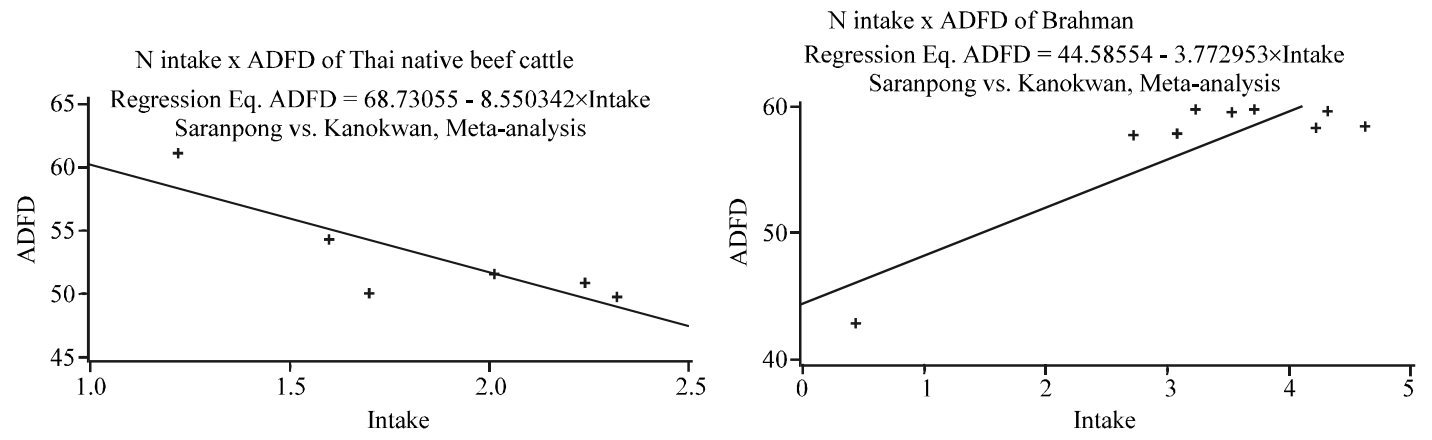

Fig. 5: Relationship between Nitrogen Intake $\left(\mathrm{NI}, \mathrm{g} \mathrm{kg}^{-1} \mathrm{BW}^{0.75}\right.$ ) and $\mathrm{ADF}$ Digestibility (ADFD\%) for Thai native beef cattle (a) describes equation: $\mathrm{ADFD}=68.73(\mathrm{SE}=5.31)-8.55(\mathrm{SE}=2.81) \mathrm{NI}\left(\mathrm{n}=6, \mathrm{R}^{2}=0.70, \mathrm{RSD}=2.63, \mathrm{p}<0.01\right)$, Brahman cattle (b) describes equation: $\mathrm{ADFD}=44.59(\mathrm{SE}=2.80)+3.77(\mathrm{SE}=0.79) \mathrm{NI}\left(\mathrm{n}=9, \mathrm{R}^{2}=0.76, \mathrm{RSD}=2.81\right.$, $\mathrm{p}<0.01)$

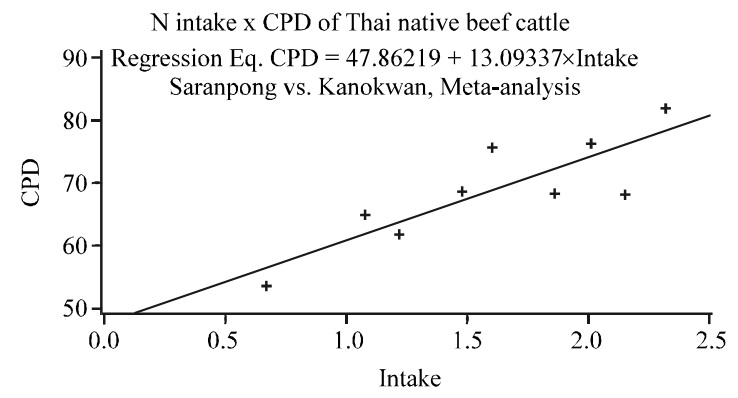

Fig. 6: Relationship between Nitrogen Intake (NI, $\mathrm{g} \mathrm{kg}^{-1}$ $\mathrm{BW}^{0.75}$ ) and CP Digestibility (CPD\%) for Thai native beef cattle (a) describes equation: $\mathrm{CPD}=47.86(\mathrm{SE}=4.50)+13.09(\mathrm{SE}=2.76) \mathrm{NI}$ $\left(\mathrm{n}=11, \mathrm{R}^{2}=0.71, \mathrm{RSD}=4.29, \mathrm{p}<0.01\right)$

$\mathrm{BUN}=7.56_{(\mathrm{SEE}=1.36)}+0.09_{(\mathrm{SE}=0.11)} \mathrm{NI}\left(\mathrm{n}=9, \mathrm{R}^{2}=0.08\right.$, $\mathrm{RSD}=2.86, \mathrm{p}<0.01)$, Brahman were $\mathrm{BUN}=14.17_{(\mathrm{SE}=3.04)}$ $1.21_{(\mathrm{SE}=0.95)} \mathrm{NI}\left(\mathrm{n}=4, \mathrm{R}^{2}=0.45, \mathrm{RSD}=2.75, \mathrm{p}<0.05\right)$ (Fig. 7).

The relationship between NI and ammonia nitrogen $\left(\mathrm{NH}_{3} \mathrm{~N}, \mathrm{mg} \%\right)$ for Thai native were $\mathrm{NH}_{3} \mathrm{~N}=-7.99_{(\mathrm{SE}=3.07)}+$ $12.14_{(\mathrm{SE}=1.84)} \mathrm{NI}\left(\mathrm{n}=7, \mathrm{R}^{2}=0.90, \mathrm{RSD}=1.56, \mathrm{p}<0.05\right)$ and the relationship between $\mathrm{NI}$ and $\mathrm{pH}$ for Thai native were $\mathrm{pH}=6.97(\mathrm{SE}=4.920 .28)-0.12_{(\mathrm{SE}=0.16)} \mathrm{NI} \quad\left(\mathrm{n}=11, \mathrm{R}^{2}=0.065\right.$, $\mathrm{RSD}=0.22, \mathrm{p}<0.01$ ) (Fig. 8 and 9). Prediction equation CP intake (CPI, $\mathrm{gCP} \mathrm{kg}^{-1} \mathrm{BW}^{0.75}$ ) with relation to average daily gain ( $\mathrm{ADG}, \mathrm{g} \mathrm{kg}^{-1} \mathrm{BW}^{0.75}$ ) was $\mathrm{CPI}=0.413+4.28$ $\left(\mathrm{R}^{2}=0.896, \mathrm{SE}=0.774, \mathrm{p}<0.05, \mathrm{n}=24\right)($ Table 4). It can be explained that the $\mathrm{CP}$ for maintenance of Thai native cattle is $4.28 \mathrm{~g} \mathrm{CP} \mathrm{kg}^{-1} \mathrm{BW}^{0.75}$. From these equations, it can be explained that the $\mathrm{CP}$ requirement for the maintenance of Thai native cattle is $4.28 \mathrm{~g} \mathrm{CP} \mathrm{kg}^{-1} \mathrm{BW}^{0.75}$.

This value was similar to Senarath et al. (2009) who reported that yearling Thai native cattle required

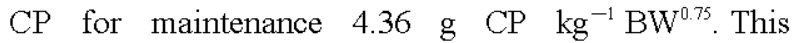
value is approximately $20.38 \%$ lower than the NRC
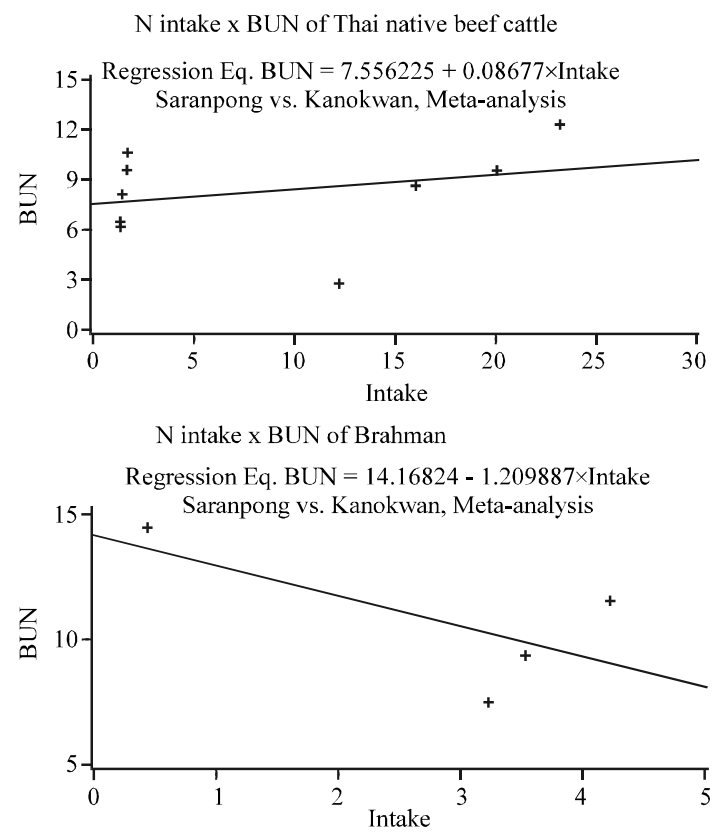

Fig. 7: Relationship between Nitrogen Intake (NI, $\mathrm{g} \mathrm{kg}^{-1}$ $\mathrm{BW}^{0.75}$ ) and Blood Urea Nitrogen (BUN, mg\%) for Thai native beef cattle (a) describes equation; $\mathrm{BUN}=7.56(\mathrm{SE}=1.36)+0.09(\mathrm{SE}=0.11) \mathrm{NI}$ $\left(\mathrm{n}=9, \mathrm{R}^{2}=0.08, \mathrm{RSD}=2.86, \mathrm{p}<0.01\right)$, Brahman cattle (b) describes equation: $\mathrm{BUN}=14.17$ $(\mathrm{SE}=3.04)-1.21(\mathrm{SE}=0.95) \mathrm{NI}\left(\mathrm{n}=4, \mathrm{R}^{2}=0.45\right.$, $\mathrm{RSD}=2.75, \mathrm{p}<0.05$ )

recommendation (5.3 $\left.\mathrm{g} \mathrm{CP} \mathrm{kg}^{-1} \mathrm{BW}^{0.75}\right)$ and was lower than Tangjitwattanachai and Sommart (2009) (5.03 g CP $\left.\mathrm{kg}^{-1} \mathrm{BW}^{0.75}\right)$ and Wilkerson et al. (1993) (5.94 $\mathrm{g} \mathrm{CP} \mathrm{kg}^{-1}$ $\mathrm{BW}^{0.75}$ ). Kearl (1982) who reported that (beef cattle $150-300 \mathrm{~kg}$ ) required $5.35-5.38$ this value is approximately $20.38 \%$ lower than the NRC recommendation (5.3 g CP $\left.\mathrm{kg}^{-1} \mathrm{BW}^{0.75}\right)$. 
Table 4: Accuracy evaluation of equations for predicted CP requirement for maintenance and gain of Thai native beef cattle and Brahman cattle

\begin{tabular}{|c|c|c|c|c|c|c|}
\hline \multirow[b]{2}{*}{ Item } & \multirow[b]{2}{*}{ Intercept } & \multirow[b]{2}{*}{ Slope } & \multirow[b]{2}{*}{ Coefficients } & \multirow[b]{2}{*}{$\mathrm{n}$} & \multicolumn{2}{|l|}{$\mathrm{p}$-value } \\
\hline & & & & & Intercept & Slope \\
\hline \multicolumn{7}{|c|}{ Thai native beef cattle } \\
\hline NI and NR & $-0.42 \pm 0.16$ & $0.58 \pm 0.10$ & 66.35 & 26 & 0.0140 & $<0.0001$ \\
\hline NI and DMD & $48.90 \pm 4.92$ & $11.10 \pm 3.02$ & 7.40 & 12 & $<0.0001$ & 0.0043 \\
\hline $\mathrm{NI}$ and $\mathrm{OMD}$ & $56.68 \pm 2.75$ & $5.27 \pm 1.72$ & 4.30 & 12 & $<0.0001$ & 0.0121 \\
\hline $\mathrm{NI}$ and NDFD & $50.94 \pm 1.16$ & $4.26 \pm 0.70$ & 1.75 & 8 & $<0.0001$ & 0.0009 \\
\hline $\mathrm{NI}$ and ADFD & $68.73 \pm 5.31$ & $-8.55 \pm 2.81$ & 4.98 & 6 & 0.0002 & 0.0383 \\
\hline $\mathrm{NI}$ and CPD & $47.86 \pm 4.50$ & $13.09 \pm 2.76$ & 6.28 & 11 & $<0.0001$ & 0.0010 \\
\hline $\mathrm{NI}$ and BUN & $7.56 \pm 1.36$ & $0.09 \pm 0.11$ & 34.35 & 9 & 0.0009 & 0.4586 \\
\hline $\mathrm{NI}$ and $\mathrm{NH}_{3} \mathrm{~N}$ & $-7.99 \pm 3.07$ & $12.14 \pm 1.84$ & 13.17 & 7 & 0.0480 & 0.0012 \\
\hline $\mathrm{NI}$ and $\mathrm{pH}$ & $6.97 \pm 0.28$ & $-0.12 \pm 0.16$ & 3.20 & 11 & $<0.0001$ & 0.4494 \\
\hline \multicolumn{7}{|c|}{ Brahman cattle } \\
\hline NI and NR & $-0.34 \pm 0.10$ & $0.70 \pm 0.04$ & 17.11 & 14 & 0.0045 & $<0.0001$ \\
\hline $\mathrm{NI}$ and DMD & $48.55 \pm 6.44$ & $7.16 \pm 1.94$ & 10.54 & 11 & $<0.0001$ & 0.0050 \\
\hline $\mathrm{NI}$ and $\mathrm{OMD}$ & $50.79 \pm 3.75$ & $6.18 \pm 1.13$ & 6.20 & 11 & $<0.0001$ & 0.0004 \\
\hline $\mathrm{NI}$ and NDFD & $51.52 \pm 2.80$ & $5.60 \pm 0.80$ & 4.00 & 9 & $<0.0001$ & 0.0002 \\
\hline $\mathrm{NI}$ and ADFD & $44.59 \pm 2.80$ & $3.77 \pm 0.79$ & 4.92 & 9 & $<0.0001$ & 0.0021 \\
\hline $\mathrm{NI}$ and BUN & $14.17 \pm 3.04$ & $-1.21 \pm 0.95$ & 25.65 & 4 & 0.0432 & 0.3313 \\
\hline
\end{tabular}

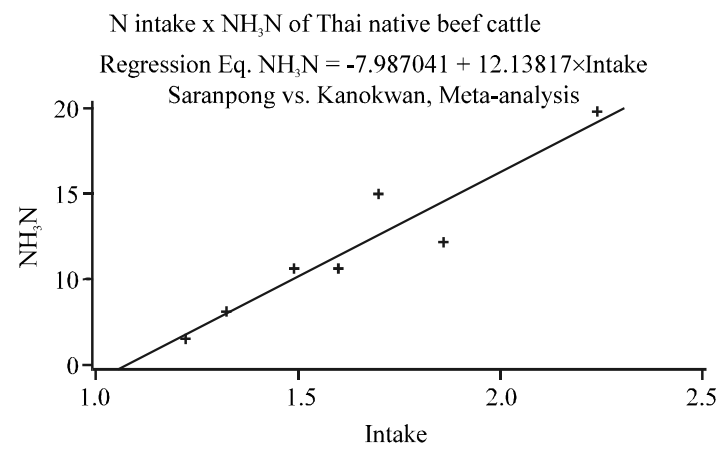

Fig. 8: Relationship between Nitrogen Intake (NI, g $\left.\mathrm{kg}^{-1} \mathrm{BW}^{0.75}\right)$ and ammonia nitrogen $\left(\mathrm{NH}_{3} \mathrm{~N}\right.$, $\mathrm{mg} \%$ ) for Thai native beef cattle (a) describes equation; $\mathrm{NH}_{3} \mathrm{~N}=-7.99(\mathrm{SE}=3.07)+$ $12.14(\mathrm{SE}=1.84) \mathrm{NI}\left(\mathrm{n}=7, \mathrm{R}^{2}=0.90, \mathrm{RSD}=1.56\right.$, $\mathrm{p}<0.05$ )

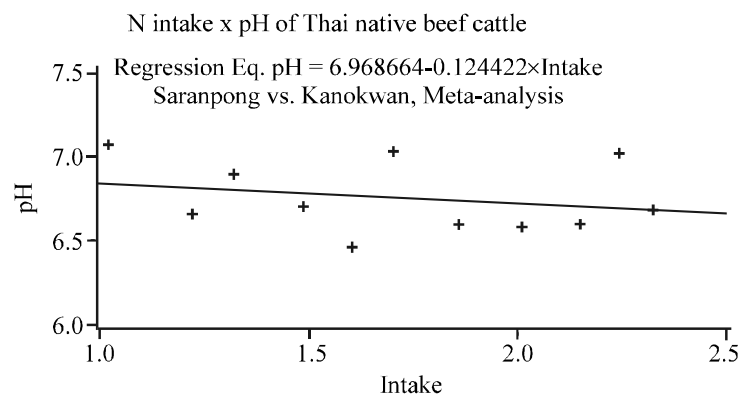

Fig. 9: Relationship between Nitrogen Intake (NI, g kg ${ }^{-1}$ $\mathrm{BW}^{0.75}$ ) and $\mathrm{pH}$ for Thai native beef cattle (a) describes equation; $\mathrm{pH}=6.97(\mathrm{SE}=4.920 .28)-0.12$ $(\mathrm{SE}=0.16) \mathrm{NI}\left(\mathrm{n}=11, \mathrm{R}^{2}=0.065, \mathrm{RSD}=0.22\right.$, $\mathrm{p}<0.01)$

\section{CONCLUSION}

In this study, nutrients digestibility and $\mathrm{ADG}$ increased $(p<0.05)$ as CP level increased. The results from this study indicate that the $\mathrm{CP}$ requirement for the maintenance of Thai native cattle is $4.28 \mathrm{~g} \mathrm{CP} \mathrm{kg}^{-1} \mathrm{BW}^{0.75}$. The results from this study were similarly to the results from meta-analysis in Thai native, Brahman cattle or in Bos indicus cattle in terms of feed intake, nutrient digestibility, ruminal $\mathrm{NH}_{3}-\mathrm{N}$ and blood urea nitrogen. If cattle in the topics are utilized more in order to exploit available low quality feed, it may contribute to the sustainable development of agriculture as well as animal production. Thai native is clearly suitable to utilize low quality roughage. Well-balanced introduction of these animals would contribute to sustainable development of not only animal production but also crop production in Thailand.

\section{ACKNOWLEDGEMENTS}

The researchers acknowledge the Suranaree University of Technology (SUT), Japan International Research Center for Agricultural Sciences (JIRCAS) and The National Research Council of Thailand (NRCT) for financial and facilities support of this research.

\section{REFERENCES}

AOAC, 1984. Official Methods of Analysis. 14th Edn., Association of Official Analytical Chemists, Washington, DC.

ARC, 1980. The Nutrient Requirements of Ruminant Livestock. Commonwealth Agricultural Bureaux, England. 
Berg, R.T. and R.M. Butterfield, 1976. New Concepts of Cattle Growth. Sydney University Press, Sydney.

Kearl, L.C., 1982. Nutrient Requirements of Ruminants in Developing Countries. 1st Edn. International Feedstuffs Institute, Utah State University, Logan, Utah, USA.

Mandal, A.B., S.S. Paul, G.P. Mandal, A. Kannan and N.N. Pathak, 2005. Deriving nutrient requirements of growing Indian goats under tropical condition. Small Ruminant Res., 58: 201-217.

NRC. (National Research Council), 2000. Nutrient Requirements of Beef Cattle. 7th Edn., National Academy Press, Washington, DC., USA.

Paul, S.S., A.B. Mandal, G.P. Mandal, A. Kannan and N.N. Pathak, 2003. Deriving nutrient requirements of growing Indian sheep under tropical condition using performance and intake data emanated from feeding trials conducted in different research institutes. Small Rumin. Res., 50: 97-107.

SAS, 1999. SAS User's Guide: Statistics. Version 8. SAS Institute Inc., Cary, NC., USA.

Schneider, B.H. and W.P. Flatt, 1975. The Evaluation of Feeds Through Digestibility Experiments. University of Georgia Press, Athens, Georgia, ISBN: 082030378X, pp: 423.
Senarath, S., K. Thummasaeng and W. Suriyapat, 2009. Protein requirement for maintenance of yearling Thai native cattle. JRCAS Working Report No.64.

St-Pierre, N.R., 2001. Invited review: Integrating quantitative findings from multiple studies using mixed model methodology. J. Dairy Sci, 84: 741-755.

Steel, R.G.D. and J.H. Torries, 1980. Principles and Procedures of Statistic a Biometereal Approach. 2nd Edn., McGraw-Hill, New York, USA.

Tangjitwattanachai, N. and K. Sommart, 2009. Protein requirements for maintenance and gain of Thai native, Brahman and Brahman crossbred beef cattle in Thailand: A meta-analysis. JRCAS Working Report No. 64, pp: 66-69, http:/www.cabdirect.org/abstracts/ $20093263170 . \mathrm{html}$.

Turner, J.W., 1980. Genetic and biological aspects of Zebu adaptability. J. Anim. Sci., 50: 1201-1205.

Van Soest, P.J., J.B. Robertson and B.A. Lewis, 1991. Methods for dietary fiber, neutral detergent fiber and nonstarch polysaccharides in relation to animal nutrition. J. Dairy Sci., 74: 3583-3597.

Wilkerson, V.A., T.J. Klopfenstein, R.A. Britton, R.A. Stock and P.S. Miller, 1993. Metabolizable protein and amino acid requirements of growing beef cattle. J. Anim. Sci., 71: 2777-2784. 